\title{
EDIFİCATE
}

I Congreso de Escuelas de Edificación y Arquitectura Técnica de España València, 4 y 5 de noviembre de 2021

Escuela Técnica Superior de Ingeniería de Edificación

Universitat Politècnica de València

Doi: https://doi.org/10.4995/EDIFICATE2021.2021.13606

\section{Movilidad de los estudiantes de Arquitectura Técnica y Edificación: diagnóstico y oportunidades}

\section{Mobility of Architectural Technology and Building Construction students: diagnosis and opportunities}

\author{
Laia Haurie Ibarra ${ }^{a}$, Chantal Alastruey Martin ${ }^{a}$ \\ aEscola Politècnica Superior d'Edificació de Barcelona, Universitat Politècnica de Catalunya \\ (laia.haurie@upc.edu), (chantal.alastruey@upc.edu)
}

\begin{abstract}
Internationalization of higher education programmes is a goal pursued by most universities and their corresponding faculties and schools. One of the main indicators to evaluate internationalization is the number of students who complete a mobility program. In this work we carry out an analysis of the evolution of the mobility of Architectural Technology and Building Construction students from the Barcelona School of Building Construction (EPSEB) since 2010 and we compare it with the evolution of mobility in the university. We analyze the main causes that limit the mobility of ATE students and we suggest actions to increase the internationalization of our studies and students.
\end{abstract}

Keywords: internationalization, mobility, Erasmus+, outgoing student.

\begin{abstract}
Resumen
La internacionalización de los estudios universitarios es una meta que persiguen la mayoría de las universidades y sus correspondientes facultades y escuelas. Uno de los principales indicadores para evaluar la internacionalización de los estudios es el número de estudiantes que realizan un programa de movilidad. En este trabajo realizamos un análisis de la evolución de la movilidad de los estudiantes de Arquitectura Técnica y Edificación (ATE) de la Escuela Politécnica Superior de Edificación de Barcelona (EPSEB) desde el año 2010 y la comparamos con la media de la universidad. Se evalúan las principales causas que limitan la movilidad de los estudiantes de ATE y se proponen actuaciones para incrementar la internacionalización de nuestros estudios y estudiantes.
\end{abstract}

Palabras clave: internacionalización, movilidad, Erasmus+, estudiante outgoing. 


\section{Introducción}

La internacionalización de los estudios universitarios es una meta que persiguen la mayoría de universidades y sus correspondientes facultades y escuelas. A nivel de las escuelas uno de los principales indicadores que se utilizan para evaluar la internacionalización es el número de estudiantes que siguen un programa de movilidad. La encuesta realizada a 1325 empresas por parte de la Agencia de la Calidad Universitaria de Catalunya (AQU), entre 2014 y 2016, revela que, junto con las prácticas en empresas, las titulaciones y estancias en el extranjero son aspectos relevantes a la hora de contratar a un titulado (AQU, 2015). En este trabajo analizamos la evolución de la movilidad de los estudiantes de Arquitectura Técnica y Edificación de la Escuela Politécnica Superior de Edificación de Barcelona (EPSEB) desde el año 2010 y la comparamos con la media de la Universitat Politècnica de Catalunya. A partir del diagnóstico inicial intentaremos proponer actuaciones para fomentar la movilidad entre nuestros estudiantes

\section{Objetivos}

En los últimos cursos desde la EPSEB hemos detectado una disminución de las solicitudes de nuestros estudiantes para realizar una estancia de movilidad. En este estudio pretendemos analizar la evolución de la movilidad de ATE desde 2010 en relación a la movilidad en la UPC y detectar las posibles causas que conllevan esta reducción. El objetivo final es tener herramientas para plantear acciones que nos permitan incrementar la internacionalización de nuestros estudiantes.

\section{Desarrollo de la innovación}

Los datos del número de estudiantes que han cursado un programa de movilidad en la EPSEB y en la UPC, así como el número de estudiantes matriculados en el grado de ATE y el total de estudiantes de grado de la UPC, se han obtenido a partir de los datos estadísticos y de gestión que publica la UPC en su web: https://gpaq.upc.edu/lldades/.

Las autoras hemos estado vinculadas durante diferentes períodos con la elaboración y gestión del plan de movilidad de la EPSEB y en este trabajo queremos compartir nuestra percepción sobre posibles causas que dificultan la internacionalización del estudiantado del grado de ATE. Asimismo, proponemos algunas ideas para promover la movilidad entre el alumnado.

Por último, hemos realizado una encuesta entre el estudiantado para conocer cómo perciben el programa de movilidad de la escuela y detectar las principales barreras que les impiden llevar a cabo una estancia en el extranjero. 


\section{Resultados}

La tabla 1 muestra la evolución del número de estudiantes en el grado de ATE y en el global de la UPC, así como el porcentaje de estudiantes que realizó una estancia de movilidad desde el curso 2010/11 hasta el curso 2020/21. Respecto al número de estudiantes podemos observar que se ha ido produciendo una reducción de estudiantes en cada curso académico, tanto en la UPC como en el grado de ATE. Esta disminución es más pronunciada en el grado de ATE, aproximadamente un $75 \%$ menos estudiantes en el curso 2020/21 respecto al curso 2010/11. En el global de la UPC el número de estudiantes se mantiene aproximadamente en un $80 \%$ de los estudiantes que había en el curso 2010/11. Es conocido que las carreras vinculadas con el sector de la construcción arrastran el efecto sobre la demanda de plazas que tuvo la crisis económica que se inició en 2008 y afectó duramente al sector de la construcción (Torre, 2014). Sin embargo, el porcentaje de estudiantes de ATE realizaron una estancia de movilidad en el extranjero (estudiantes outgoing) creció coincidiendo con los años de crisis económica, situándose en el curso $2014 / 15$ en $5,6 \%$ por encima del global de la UPC (4,1\%). A partir de ese momento hemos ido observando una disminución del interés del estudiantado por la movilidad con una reducción hasta el $1,2 \%$ de estudiantes outgoing en el curso $2018 / 19$, todavía no afectado por la pandemia.

Tabla 1. Evolución del número de estudiantes que han realizado una estancia de movilidad, en el grado de ATE y en el global de la UPC.

\begin{tabular}{|c|c|c|c|c|c|c|}
\hline \multirow{2}{*}{$\begin{array}{c}\text { Curso } \\
\text { académico }\end{array}$} & \multicolumn{2}{|c|}{ Estudiantes grado } & \multicolumn{2}{|c|}{ Estudiantes outgoing } & \multicolumn{2}{|c|}{$\begin{array}{l}\% \text { Estudiantes } \\
\text { outgoing }\end{array}$} \\
\hline & ATE & UPC & ATE & UPC & ATE & UPC \\
\hline $2020 / 21$ & 577 & 25901 & 2 & 787 & 0,3 & 3,0 \\
\hline $2019 / 20$ & 677 & 25378 & 4 & 1328 & 0,6 & 5,2 \\
\hline $2018 / 19$ & 657 & 27951 & 8 & 1337 & 1,2 & 4,8 \\
\hline $2017 / 18$ & 736 & 27966 & 20 & 1507 & 2,7 & 5,4 \\
\hline $2016 / 17$ & 794 & 28707 & 24 & 1401 & 3,0 & 4,9 \\
\hline $2015 / 16$ & 1019 & 28902 & 34 & 1353 & 3,3 & 4,7 \\
\hline $2014 / 15$ & 1241 & 30387 & 69 & 1249 & 5,6 & 4,1 \\
\hline $2013 / 14$ & 1526 & 30681 & 68 & 1646 & 4,5 & 5,4 \\
\hline $2012 / 13$ & 1837 & 31214 & 92 & 2613 & 5,0 & 8,4 \\
\hline $2011 / 12$ & 2165 & 31882 & 71 & 2150 & 3,3 & 6,7 \\
\hline $2010 / 11$ & 2330 & 32698 & 75 & 2243 & 3,2 & 6,9 \\
\hline
\end{tabular}

A continuación, analizaremos las principales razones que pueden explicar esa falta de interés por la movilidad y qué acciones podemos llevar a cabo para intentar revertir esa tendencia. 


\section{Percepción de la movilidad}

Como se ha podido ver en la evolución de la movilidad en los últimos 10 años, esta ha ido empeorando, en número, conforme la crisis económica iba remitiendo. Es decir, durante los primeros años, la movilidad se percibía como una oportunidad que tenían nuestros estudiantes para poder abrirse paso en otros mercados laborales menos perjudicados por la crisis del sector. El estudiantado se iba de Erasmus con la idea, en muchos casos, de no volver y aprovechar su estancia para encontrar un futuro que en nuestro país se veía, laboralmente hablando, complicado.

Sin embargo, curiosamente esto no dio valor a la movilidad en sí, el período de estancia en el extranjero no se percibió como una oportunidad de adquirir nuevas competencias, que fuesen un valor diferencial en el momento de entrar en el mercado laboral como arquitectos técnicos y arquitectas técnicas.

¿A qué se debe esto? Por una parte, a la imagen que tiene la propia movilidad Erasmus, lamentablemente más famosa por su componente lúdico que por los logros académicos de los que participan en este programa. Por otra parte, parece que el sector de la construcción históricamente siempre ha sido un sector fuertemente vinculado al territorio. Así, en la mayoría de los casos y exceptuando multinacionales, que en muchas ocasiones son de origen extranjero, las empresas constructoras trabajan principalmente en territorio nacional.

Esta fuerte relación de la territorialidad de las empresas constructoras ha provocado que nuestro estudiantado no vea la necesidad, ni siquiera la utilidad, de realizar una estancia de movilidad en el extranjero, puesto que la experiencia internacional no se percibe como algo que será valorado en el momento de entrar en el mercado laboral.

Si nuestro estudiantado percibe que en la mayoría de los casos no va a necesitar la experiencia internacional y, es más, que esa experiencia no se va a valorar en su currículo como una adquisición de competencias, sino más bien como una estancia lúdica, ¿querrá participar en una movilidad cuando tiene a su alcance otras posibilidades que se perciben cómo más útiles?

Por ello y tras ver que la realización de prácticas en empresa, que además en la UPC están remuneradas, se percibe como algo más útil y que interesa más a nuestro estudiantado, se ha planteado la posibilidad de facilitar al estudiantado la realización de Erasmus prácticas. Se pretende tener una bolsa de prácticas internacionales a la que el estudiantado interesado pueda acceder para presentar su candidatura.

\section{Coste económico de la movilidad}

Cómo bien sabemos el coste de vida en otros países es superior al nuestro, aunque hay que decir que Barcelona, ciudad donde está situada nuestra escuela, es considerada una ciudad cara con respecto a otras. Aún así, tradicionalmente el estudiantado no abandona el hogar familiar durante sus estudios, lo que permite que, en la mayoría de casos, aunque el coste de vida sea elevado, la vivienda y manutención del estudiante no sea un factor que impida la realización de una carrera universitaria. 
Sin embargo, esa ventaja se pierde al realizar una movilidad y el coste de vida del estudiante, durante su estancia en el extranjero, en muchos casos se convierte en un coste inasumible para las familias.

Hay que decir que las movilidades Erasmus van asociadas a una ayuda económica mensual, pero esta ayuda no cubre el coste de vida de la mayoría de países europeos.

Este es un problema al que difícilmente se le pueda encontrar solución por parte de la Escuela o la Universidad. Pero sí se ha observado en los grupos de trabajo con estudiantado, que estos desconocen que además de la ayuda económica Erasmus, puede solicitar otras becas compatibles con este tipo de movilidades (de la Generalitat de Catalunya y de otras entidades privadas).

Es por ello que se realizará una campaña de comunicación para hacer llegar esta información al estudiantado, no sólo la información debe estar disponible en el apartado de movilidad de la web, puesto que sólo la verían aquellos que entrasen en ese apartado, sino que es imprescindible que todos nuestros estudiantes matriculados sepan qué es una movilidad, qué tipos de movilidades hay, a qué países, qué requisitos hay para poder realizarlas y qué ayudas económicas pueden conseguir en cada caso. Además, también hay que difundir los beneficios que realizar una estancia en extranjero puede aportar, tanto en lo que se refiere a la adquisición de competencias, al aprendizaje de una tercera lengua y a la formación de una red de contactos internacional que puede ser muy beneficiosa para el futuro laboral de nuestro estudiantado.

Esta campaña debería ir también acompañada de la presentación de casos de antiguos alumnos que nos hablen de su experiencia durante la movilidad $y$, sobre todo, de qué beneficios le ha aportado una vez finalizados sus estudios.

\section{Oferta de movilidad}

La UPC es una universidad donde la gestión de los acuerdos de movilidad Erasmus europeos está totalmente descentralizada, es decir, cada escuela o facultad gestiona sus propios acuerdos bilaterales. Si a esto le sumamos que en la UPC ya existen grados como el de Ingeniería Civil y Arquitectura, vemos como en muchas ocasiones se nos complica el poder realizar acuerdos con universidades de prestigio, sobre todo en países donde no hay una correspondencia directa con el grado en Arquitectura Técnica y Edificación.

¿Qué necesidad tiene una universidad europea de prestigio en hacer un acuerdo bilateral de movilidad con nuestro grado, si ya los tiene con Arquitectura e Ingeniería Civil?, ¿Es más qué sentido tiene para ellos hacer ese acuerdo si en su país no existe un grado de building engineering o similar? $Y$ ¿qué podemos hacer con esos países donde sí existe una carrera similar pero es una carrera técnica de 2 o 3 años, una minor?

No hay que olvidar que nuestro grado es habilitante y que no podemos aceptar que la realización de una estancia en el extranjero perjudique a nuestro estudiantado no permitiendo que adquieran las competencias necesarias para acabar de forma adecuada en tiempo y forma sus estudios. Este es un compromiso adquirido no sólo con nuestro 
estudiantado, sino también, como universidad pública que somos, con la sociedad a la que prestamos servicio.

Todas estas consideraciones nos han hecho replantearnos nuestra oferta de movilidad y buscar qué podemos ofrecer para ser más atractivos ante futuros partners. Partners que deben ser universidades de reconocido prestigio, que sepamos que pueden aportar a nuestro estudiantado una experiencia académica rica, que le sirva para su futuro profesional.

Tras trabajar con grupos de estudiantes propios y también con estudiantes de movilidad incoming vimos que nuestros principales puntos fuertes eran, por una parte el prestigio de la escuela y la universidad, por otra nuestra cada vez más amplia oferta de asignaturas en inglés, además el prestigio de algunos de nuestros laboratorios, referentes en investigación en ámbitos como la prevención contra incendios, los materiales de construcción, la eficiencia energética y la rehabilitación de patrimonio construido y, finalmente, la ciudad, Barcelona.

Teniendo estas ventajas presentes se ha trabajado en ampliar la oferta de asignaturas en inglés de modo que actualmente ya se puede hacer 60 ECTS en ese idioma. Esto nos ha hecho crecer en los últimos años, dado que hemos pasado de recibir 40 alumnos de movilidad de procedencia internacional en el curso 2012/2013 a 74 en el curso 2018/2019, siendo 52 en el 2019/2020 curso en que la pandemia impidió seguir creciendo (GPAQUPC,2021).

También se está trabajando para dar mayor visibilidad a actividades que, si bien ya se llevaban a cabo, nunca habían tenido protagonismo en nuestra web y redes sociales. Así cada vez que se hacen visitas a edificios de alto valor arquitectónico, como el recinto modernista del Hospital de la Santa Creu i Sant Pau o la Sagrada familia entre muchos otros, se hacen sus correspondientes publicaciones.

Hay que reconocer que tenemos una gran ventaja respecto otros países y poblaciones al estar en una ciudad con un patrimonio arquitectónico de gran valor, que atrae cada año a miles de turistas, ese es uno de los principales motivos por lo que resultamos atractivos a nuestro estudiantado internacional. Así que podemos sacarle provecho haciendo que ese patrimonio forme parte de la experiencia del estudiantado internacional. Curiosamente era algo que ya hacíamos, pero de nuevo no habíamos sido conscientes de la necesidad de dar visibilidad a este tipo de actividades.

Y como ya se ha dicho también están nuestros laboratorios, referente en ámbitos de investigación y transferencia de tecnología y que hasta ahora no han tenido el protagonismo debido. Estos laboratorios no están desvinculados de la docencia, al contrario, todos ellos participan de forma directa en la docencia del grado, haciendo diversas clases, a lo largo del curso, en sus instalaciones. Esto nos permite no tener que cambiar la forma como trabajamos, sino simplemente, tener que dar visibilidad a algo que, si bien para nosotros forma parte de la normalidad, para otros estudiantes de otras procedencias resulta tremendamente atractivo. Además, a esto hay que añadir, que nuestro profesorado, 
muchos de ellos y ellas vinculados a estos laboratorios, participa en proyectos europeos de investigación y fomento de la docencia, con lo que tiene socios en distintas instituciones.

Finalmente hay un último punto que también nos puede ayudar a mejorar nuestra oferta de movilidad, y es las redes internacionales en las que participa la UPC, como se puede ver en esta imagen, pertenecemos a una universidad muy activa en el ámbito de la internacionalización.

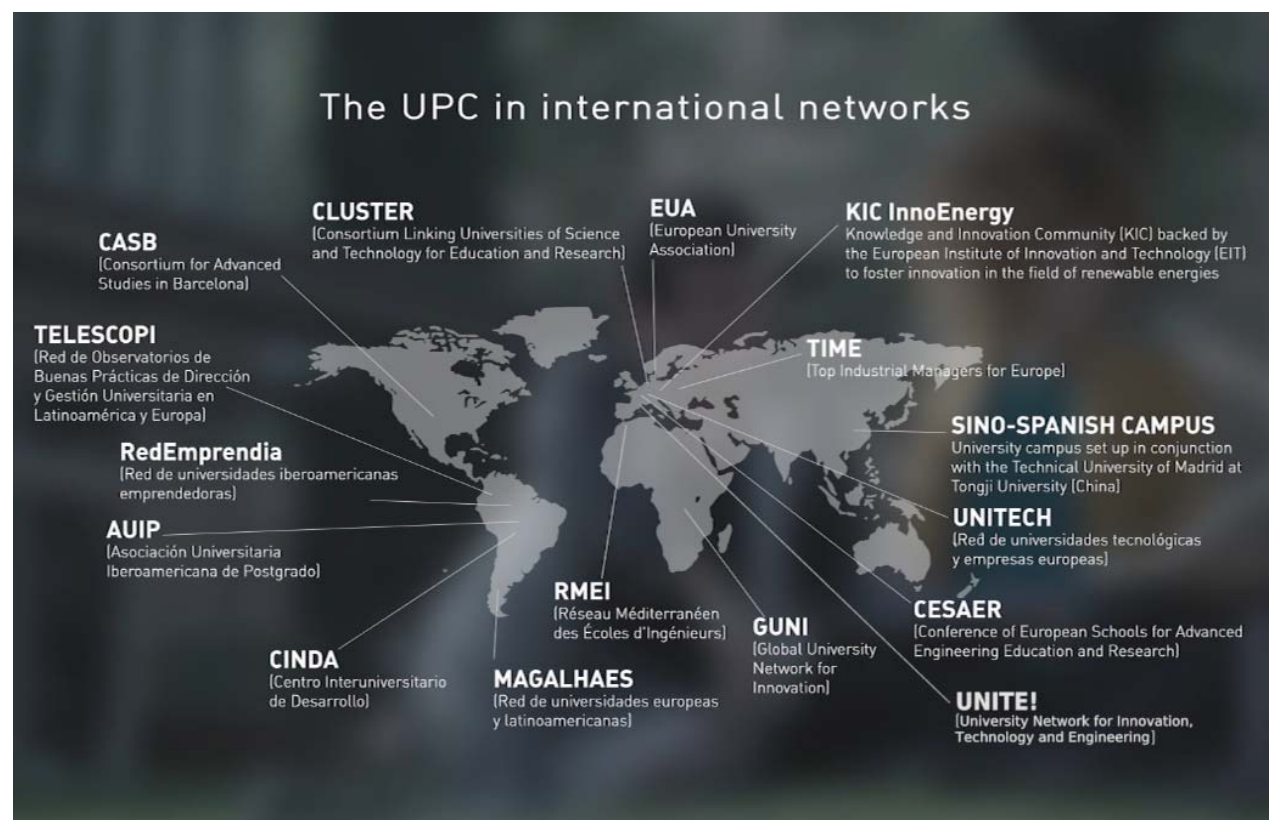

Fig. 1 Redes internacionales en las que participa la UPC (GRI-UPC, 2021)

Ahora que sabemos qué podemos aportar a nuestros futuros partners, ha llegado el momento de, con la ayuda del gabinete de relaciones internacionales de la UPC, contactar con universidades que ya son socias y que pueden ver en nosotros, una escuela más pequeña que las que ofrecen los grados de arquitectura e ingeniería civil, un espacio que cuida mucho su oferta de estudios en inglés y donde además sus estudiantes podrán participar en actividades altamente enriquecedoras, tanto aprendiendo del patrimonio arquitectónico de la ciudad, como de las actividades de experimentación en laboratorios que están vinculadas a distintas asignaturas y talleres del grado.

\section{Conclusiones}

Tras el análisis realizado hemos identificado diversos factores que dificultan el acceso a la movilidad del estudiantado de ATE y planteamos las siguientes líneas de actuación para fomentar la internacionalización del alumnado: 
- Ampliar la oferta de movilidad a estancias cortas pensadas para ser realizadas fuera del período lectivo y que tengan como objetivo la adquisición de competencias concretas muy relacionadas con el temario del plan de estudios.

Para poder ofrecer estas estancias cortas tenemos previsto aprovechar que el nuevo programa Erasmus ya contempla este tipo de estancias, que la Universitat Politècnica de Catalunya (UPC) pertenece a la red de universidades europea Unite! que organiza, entre otras muchas cosas, la oferta de cursos de varias semanas, y también la participación de profesorado de la escuela en proyectos Erasmus+ dentro de las Key Action 2 que contemplan y permiten financiar la movilidad de estudiantes para realizar cursos intensivos.

- Mejorar la oferta de movilidad. Ahora mismo tenemos una oferta variada, pero según nos han notificado representantes de los estudiantes en distintos grupos de trabajo, esta oferta no es lo suficientemente atractiva.

- Potenciar el Erasmus prácticas. Según hemos podido constatar las prácticas en empresas sí están muy bien valoradas por nuestro estudiantado y, de hecho, exceptuando los dos años de pandemia, el número total de convenios por curso, estaba subiendo cada año. Sin embargo, nuestro estudiantado no realiza ningún Erasmus prácticas, puesto que, según nos han expresado, ven muy complicado conseguir esas prácticas en el extranjero sin tener contactos previos a los que poder acudir. Por ello, aprovechando los contactos que tiene la escuela y su profesorado, queremos facilitar una oferta de prácticas en otros países que ayude a nuestro estudiantado a adquirir más experiencia en un mundo cada vez más globalizado.

- Hacer una campaña de comunicación para acercar todas las posibilidades para realizar una movilidad y sus beneficios al estudiantado. No solo hay que facilitar la información a aquellos que la busquen, ahora mismo es necesario acercar esta información a toda la comunidad, puesto que hemos detectado mucho desconocimiento respecto a qué posibilidades tienen y cómo realizar las distintas posibilidades de movilidad que ofrece la universidad.

\section{Referencias}

AGÈNCIA PER A LA QUALITAT DEL SISTEMA UNIVERSITARI DE CATALUNYA (AQU) (2015). Ocupabilitat i competències dels graduats recents: l'opinió d'empreses i institucions. Barecelona: AQU. <https://www.aqu.cat/doc/doc_69192241_1.pdf> [Consulta: 30 de junio de 2021]

GABINET DE PLANIFICACIÓ, AVALUACIÓ I QUALITAT (GPAQ) - UPC. Dades estadístiques i de gestió. <https://gpaq.upc.edu/lldades/indicador.asp?index=1_6_1> [Consulta: 30 de junio de 2021]

GABINETE DE RELACIONES INTERNACIONALES (GRI) - UPC. Alianzas internacionales. $<$ https://www.upc.edu/sri/es/alianzas> [Consulta: 30 de junio de 2021] 
Movilidad de los estudiantes de Arquitectura Técnica y Edificación: diagnóstico y oportunidades Mobility of Architectural Technology and Building Construction students: diagnosis and opportunities

(TORRE, 2014) TORRE, N (2014). "Las carreras ligadas a la crisis de la construcción se hunden en las aulas" en El Mundo.

https://www.elmundo.es/comunidad-valenciana/2014/07/19/53ca2073e2704efb4c8b456e.html [Consulta: 30 de junio de 2021] 